\title{
ANDES

www.scielo.cl

\section{Efecto de la normalización de TSH y T4 libre sobre el perfil lipídico en población pediátrica con hipotiroidismo primario}

\author{
Effect of the normalization of TSH and free T4 on lipid profile in a pediatric \\ population with primary hypothyroidism
}

\author{
Aleida Rivera-Hernández ${ }^{\mathrm{a}}$, Raúl Rojas-Martínez ${ }^{\mathrm{b}}$, Victoria Mendoza-Zubieta $^{\mathrm{c}}$, Lourdes Balcázar-Hernández ${ }^{\mathrm{a}, \mathrm{c}}$ \\ aDepartamento de Endocrinología Pediátrica. Hospital de Pediatría. Unidad Médica de Alta Especialidad, CMN Siglo XXI, IMSS. Ciudad de México, \\ México \\ bUnidad de Posgrado, Escuela Superior de Medicina. Instituto Politécnico Nacional. Ciudad de México, México \\ ‘Departamento de Endocrinología. Hospital de Especialidades. Unidad Médica de Alta Especialidad, CMN SXXI, IMSS. Ciudad de México, México
}

Recibido: 4 de junio de 2020; Aceptado: 22 de septiembre de 2020

¿Qué se sabe del tema que trata este estudio?

El hipotiroidismo primario se asocia con un aumento del riesgo cardiovascular y dislipidemia. En población adulta, el tratamiento con levotiroxina ha demostrado una mejoría en parámetros cardiometabólicos, incluyendo el perfil lipídico, siendo escasos los datos en población pediátrica.
¿Qué aporta este estudio a lo ya conocido?

En niños con hipotiroidismo existe una asociación entre TSH, T4L y el perfil lipídico. La normalización del perfil tiroideo permite una reducción de colesterol, triglicéridos y LDL-C. La dislipidemia persistente post-tratamiento se asocia con obesidad, sobrepeso y dislipidemia pretratamiento.

\section{Resumen}

El hipotiroidismo ha sido asociado con dislipidemia. Su tratamiento con levotiroxina ha mostrado un efecto positivo sobre el perfil lipídico en adultos, siendo poco descrito en población pediátrica. Objetivo: Evaluar el efecto de la normalización del perfil tiroideo sobre el perfil lipídico en niños con hipotiroidismo primario. Pacientes y Método: Estudio retrospectivo en niños de 6 a 16 años de edad, con diagnóstico de hipotiroidismo primario por tiroiditis de Hashimoto, en tratamiento con levotiroxina, y que contaron con evaluación de lípidos séricos antes y durante su tratamiento. Se evaluó el perfil lipídico en 2 tiempos: el primero, referido como "antes del tratamiento con levotiroxina” (al diagnóstico de hipotiroidismo primario) y el segundo, referido como "a la normalización del perfil tiroideo". Se registraron datos sociodemográficos y antropométricos. La evaluación del perfil lipídico consistió en la determinación sérica de colesterol total (CT), colesterol de alta densidad (HDL-C) y TG. El fenotipo de las dislipidemias se caracterizó a través de la clasificación de Fredrickson. Resultados: Se incluyeron 72 pacientes (61\% mujeres; edad de $11,5 \pm 2,9$ años), de los cuales 58,3\% $(\mathrm{n}=42)$ presentó dislipidemia pretratamiento. En estado hipotiroideo, se evidenció la correlación de TSH con
Palabras clave: Hipotiroidismo; Tiroiditis de Hashimoto; Levotiroxina; Dislipidemia

Correspondencia:

Balcázar-Hernández Lourdes

dra.lourdesbalcazar@gmail.com 
CT $(r=0,36 ; p=0,002)$, LDL-C $(r=0,46 ; p=0,01)$ y HDL-C $(r=-0,33 ; p=0,004)$. A la normalización del perfil tiroideo se evidenció la reducción de CT [184 mg/dL (RIC 92-322) vs 147 mg/dL (RIC 92-283); $\mathrm{p}=0$ 0,05], LDL-C [99 mg/dL (RIC 44-232) vs $82 \mathrm{mg} / \mathrm{dL}$ (RIC 41-168); $\mathrm{p}=0,02$ ], TG [113 $\mathrm{mg} / \mathrm{dL}$ (RIC 50-483) vs $88 \mathrm{mg} / \mathrm{dL}$ (RIC 16-343); $\mathrm{p}=0,03]$ y de la frecuencia de dislipidemia [58,3\% vs 22,2\%; $\mathrm{p}=0,001)$, así como la corrección de CT con TG $(\mathrm{r}=0,35 ; \mathrm{p}=0,02)$ y LDL-C $(\mathrm{r}=0,88$; $\mathrm{p}=0,01)$. La persistencia de dislipidemia se asoció con obesidad $(\mathrm{r}=0,27 ; \mathrm{p}=0,02)$, sobrepeso $(\mathrm{r}=0,58 ; \mathrm{p}=0,001)$ y dislipidemia pretratamiento $(\mathrm{r}=0,53 ; \mathrm{p}=0,001)$. Conclusiones: Existe una asociación entre TSH, CT, LDL-C y HDL-C en hipotiroidismo. Al normalizarse el perfil tiroideo, se evidenció una reducción de CT, TG, LDL-C y frecuencia de dislipidemia. La dislipidemia persistente post-tratamiento se asoció con obesidad, sobrepeso y dislipidemia pretratamiento.

\begin{abstract}
Hypothyroidism has been associated with dyslipidemia. Its treatment with levothyroxine has shown a positive effect on the lipid profile in adults, however, there is a lack of data on the pediatric population. Objective: to evaluate the effect of the thyroid profile normalization on the lipid profile in children with primary hypothyroidism. Patients and Method: Retrospective study in children aged from 6 to 16 years, with diagnosis of primary hypothyroidism due to Hashimoto's thyroiditis, in treatment with levothyroxine, and who had an evaluation of serum lipids before and during their treatment. The lipid profile was evaluated in 2 stages: the first one referred to as "before levothyroxine treatment" (at the diagnosis of primary hypothyroidism) and the second one referred to as " thyroid profile normalization" (when normalization of Thyroid-stimulating hormone [TSH] and free T4 [FT4] was achieved during levothyroxine treatment). Sociodemographic and anthropometric data were recorded. The lipid profile evaluation consisted of the serum determination of total cholesterol (TC), high-density cholesterol (HDL-C), and TG. The phenotype of dyslipidemias was determined according to the Fredrickson's classification. Results: 72 patients were included (61\% women; age $11.5 \pm 2.9$ years $)$, out of which $58.3 \%(\mathrm{n}=42)$ presented pre-treatment dyslipidemia. In hypothyroid state, it was evident the correlation of TSH with TC $(r=0.36 ; p=0.002)$, LDL-C $(r=0.46 ; p=0.01)$, and HDL-C $(\mathrm{r}=-0.33 ; \mathrm{p}=0.004)$. The thyroid profile normalization showed the reduction of TC [184 mg/dL (IQR 92-322) vs 147 mg/dL (IQR 92-283); p = 0.05], LDL-C [99 mg/dL (IQR 44-232) vs $82 \mathrm{mg} / \mathrm{dL}$ (IQR 41-168); $\mathrm{p}=0.02$ ], TG [113 mg/dL (IQR 50-483) vs $88 \mathrm{mg} / \mathrm{dL}$ (IQR 16-343); $\mathrm{p}=0.03$ ], and the frequency of dyslipidemia [58.3\% vs 22.2\%; $\mathrm{p}=0.001$ ), as well as the TC correction with TG $(\mathrm{r}=0.35 ; \mathrm{p}=0.02)$ and LDL-C $(\mathrm{r}=0.88 ; \mathrm{p}=0.01)$. Persistent dyslipidemia was associated with obesity $(\mathrm{r}=0.27 ; \mathrm{p}=0.02)$, overweight $(\mathrm{r}=0.58 ; \mathrm{p}=0.001)$, and pre-treatment dyslipidemia $(\mathrm{r}=0.53 ; \mathrm{p}=0.001)$. Conclusions: There is an association between TSH, TC, LDL-C, and HDL-C in hypothyroidism. When the thyroid profile was normalized, there was a reduction of TC, TG, LDL$\mathrm{C}$, and dyslipidemia frequency. Persistent dyslipidemia was associated with obesity, overweight, and pre-treatment dyslipidemia.
\end{abstract}

\section{Keywords:}

Hypothyroidism;

Hashimoto Thyroiditis;

Levothyroxine;

Dyslipidemia

\section{Introducción}

Las hormonas tiroideas tienen un papel fundamental en la homeostasis de lípidos al regular la actividad de receptores, enzimas y proteínas de transferencia involucrados en el metabolismo de lipoproteínas ${ }^{1}$. En niños y adolescentes, la tiroiditis de Hashimoto representa la causa más frecuente de hipotiroidismo primario adquirido, siendo más prevalente durante el inicio de la pubertad e infrecuente antes de los 3 años de edad. La tiroiditis de Hashimoto muestra una predominancia en el sexo femenino (relación 3-4:1) y puede estar asociada con otras enfermedades autoinmunes, síndrome de Down o síndrome de Turner. El espectro de su presentación es amplio, cursando desde un estado asinto- mático hasta coma mixedematoso ${ }^{2}$. En pacientes con hipotiroidismo primario por tiroiditis de Hashimoto se ha evidenciado un aumento de marcadores proaterogénicos, dislipidemia y riesgo cardiovascular ${ }^{3}$. Las alteraciones del metabolismo de lípidos por hipotiroidismo se deben a la deficiencia de hormonas tiroideas. Dentro de los mecanismos fisiopatológicos relacionados se encuentran: la disminución en la expresión de hidroximetilglutaril Co-A reductasa ${ }^{4}$, la reducción de receptores superficiales de LDL-C $\mathrm{C}^{5}$, el aumento en la absorción intestinal de colesterol ${ }^{6}$, alteraciones en el transporte reverso del colesterol $^{7-9}$, alteraciones en la formación y excreción fecal de ácidos biliares ${ }^{10} \mathrm{y}$ disminución en la actividad de lipoproteínlipasa ${ }^{8}$.

El tratamiento sustitutivo con levotiroxina ha de- 
mostrado una mejoría en parámetros cardiometabólicos, incluyendo el perfil lipídico. La mayoría de los estudios se han realizado en población adulta, siendo escasos en población pediátrica, y más aún, en población latina ${ }^{11-14}$.

El objetivo de nuestro estudio fue evaluar el efecto de la normalización de Hormona Tiro-estimulante (TSH) y T4 libre (T4L) sobre el perfil lipídico y sus alteraciones en población pediátrica con hipotiroidismo primario por tiroiditis de Hashimoto tratados con levotiroxina, así como determinar la asociación entre TSH, T4L, IMC y el perfil lipídico antes del tratamiento con levotiroxina y a la normalización del perfil tiroideo.

\section{Pacientes y Método}

Estudio retrospectivo en niños con hipotiroidismo primario atendidos en el Hospital de Pediatría, Centro Médico Nacional Siglo XXI, IMSS, Ciudad de México, de enero de 2011 a junio de 2017. El estudio fue revisado y aprobado por el Comité Local de Ética e Investigación en Salud del Hospital de Pediatría, Centro Médico Nacional Siglo XXI, IMSS. Los datos fueron obtenidos a través de la revisión de expedientes médicos.

Se incluyeron mujeres y hombres entre 6 y 16 años de edad, con diagnóstico de hipotiroidismo primario por tiroiditis de Hashimoto, tratamiento con levotiroxina y que contaron con la evaluación bioquímica de lípidos séricos antes y durante su seguimiento médico. El hipotiroidismo primario por tiroiditis de Hashimoto fue definido como la producción deficiente de hormonas tiroideas causada por una alteración funcional de la glándula tiroides mediada por anticuerpos, diagnosticado bioquímicamente ante concentraciones séricas bajas de T4L (valor de referencia: 0,93-1,7 ng/ $\mathrm{dL}$ ), aumento de TSH (valor de referencia: 0,27-4,2 $\mu \mathrm{IU} / \mathrm{mL}$ ) y la presencia de anticuerpos anti-peroxidasa tiroidea $\geqq 9,0 \mathrm{IU} / \mathrm{mL}$ y/o anti-tiroglobulina $\geqq 4,0 \mathrm{IU} /$ $\mathrm{mL}$. Se excluyeron pacientes con diagnóstico de diabetes mellitus, dislipidemia primaria, síndrome nefrótico, hipotiroidismo congénito, secundario a procedimientos, central o subclínico, uso de glucocorticoides, enfermedad autoinmune no tiroidea, neoplasias, enfermedad renal, hepática u otra enfermedad crónicodegenerativa, inadecuado apego al tratamiento con levotiroxina, síndrome de malabsorción o aquellos en los que no se logró la normalización de TSH y T4 libre. La normalización de TSH y T4L fue definida como el logro en las concentraciones de TSH entre 0,27 y 4,2 $\mu \mathrm{IU} / \mathrm{mL}$ y de T4L entre 0,9 y $1,7 \mathrm{ng} / \mathrm{dL}$, medidos por inmunoanálisis electroquimioluminiscente (ECLIA, Cobas, Roche Diagnostics $\left.{ }^{\circledR}\right)$. La dosis administrada de levotiroxina fue: 4 a $5 \mu \mathrm{g} / \mathrm{kg} /$ día entre los 6 y 12 años de edad, 2 a $3 \mu \mathrm{g} / \mathrm{kg} /$ día en pacientes mayores de 12 años con desarrollo o pubertad incompletos y 1,6 $\mu \mathrm{g} / \mathrm{kg} /$ día en mayores de 12 años con desarrollo y pubertad completos. Para fines del estudio, se normaron 2 tiempos: el primero, referido como "antes del tratamiento con levotiroxina" (al diagnóstico de hipotiroidismo primario) y el segundo, referido como "a la normalización del perfil tiroideo" (al lograrse la normalización de TSH y T4 libre durante el tratamiento con levotiroxina). Se recabaron datos sociodemográficos y antropométricos (edad, sexo, estadio Tanner, peso, talla, Z-score para peso y talla, e IMC), determinándose sobrepeso con un IMC entre el percentil 85-94 y obesidad con IMC en percentil 95 o mayor ${ }^{15}$. La evaluación del perfil lipídico consistió en la determinación sérica, tras ayuno de 12 horas, de las concentraciones de colesterol total (CT), colesterol de alta densidad (HDL-C) y TG, mediante colorimetría (Cobas Lipid Panel, Roche Diagnostics ${ }^{\circledR}$ ), antes del tratamiento con levotiroxina y a la normalización del perfil tiroideo. Las concentraciones de colesterol de baja densidad (LDL-C) se calcularon a través de la fórmula de Friedewald $(\mathrm{LDL}=$ Colesterol total [triglicéridos/5] - HDL-Colesterol), siempre y cuando las concentraciones de TG se encontraran por debajo de $400 \mathrm{mg} / \mathrm{dL}^{16}$. De acuerdo a la National Cholesterol Education Program (NCEP), se determinó hipertrigliceridemia ante concentraciones de TG $>100 \mathrm{mg} / \mathrm{dL}$ en menores de 9 años y TG $>130 \mathrm{mg} / \mathrm{dL}$ entre los 10 y 19 años, hipercolesterolemia ante concentraciones de CT $\geq 200 \mathrm{mg} / \mathrm{dL}$, "elevación de LDL" ante concentraciones de LDL-C $\geq 130 \mathrm{mg} / \mathrm{dL}$ y "HDL bajo" ante concentraciones de HDL-C $<40 \mathrm{mg} / \mathrm{dL}^{17}$. El fenotipo de las alteraciones del perfil lipídico (dislipidemia)se caracterizó a través de la clasificación de Fredrickson ${ }^{18}$.

\section{Análisis estadístico}

Las variables cualitativas fueron descritas en frecuencias y proporciones. Las variables cuantitativas fueron reportadas en media y desviación estándar (DS) o mediana y rango intercuartílico (RIC) de acuerdo a su distribución. La normalidad se determinó con la prueba de Shapiro-Wilks. Para la comparación de variables cualitativas se utilizó la prueba Chi cuadrado, así como Wilcoxon y U Mann-Whitney para variables cuantitativas, y para establecer asociaciones entre las variables se utilizó el coeficiente de correlación de Spearman. Se determinó significancia estadística ante un valor de $\mathrm{p}<0,05$. Como herramientas estadísticas se utilizaron los programas SPSSv.13 y GraphPad Prism.

\section{Resultados}

\section{Pacientes}

Se incluyó un total de 72 pacientes con una media de edad de 11,5 $\pm 2,9$ años, de los cuales el $61 \%(n=44)$ fueron mujeres. De acuerdo al estadio Tanner, el 68\% 
$(\mathrm{n}=49)$ fueron púberes [estadio II: $18 \%(\mathrm{n}=13)$; estadio III: $14 \%(\mathrm{n}=10)$ y estadio IV: $14 \%(\mathrm{n}=10)]$. En relación al estado nutricional, 4,2\% $(\mathrm{n}=3)$ tuvo peso bajo, $43 \%(n=31)$ peso normal, $25 \%(n=18)$ sobrepeso y $27,8 \%(n=20)$ obesidad. Antes del tratamiento con levotiroxina, se evidenciaron concentraciones de TSH de 29,8 $\mu \mathrm{IU} / \mathrm{mL}$ (RIC 16,7-69,8) y T4L de 0,55 ng/ dL (RIC 0,35-0,69).

\section{Alteraciones del perfil lipídico y caracterización del fenotipo en pacientes con hipotiroidismo primario}

Antes del tratamiento con levotiroxina, los componentes del perfil lipídico mostraron las siguientes concentraciones: CT $184 \mathrm{mg} / \mathrm{dL}$ (RIC 92-322), LDL-C 99 $\mathrm{mg} / \mathrm{dL}$ (RIC 44-232), HDL-C 48 mg/dL (RIC 22-80) y TG 113 mg/dL (RIC 50-483). El 58,3\% (n = 42) de los pacientes presentó dislipidemia. Se evidenció hipercolesterolemia en un $43 \%(n=31)$, hipertrigliceridemia en un $36 \%(n=26)$, elevación de LDL en un $50 \%$ $(\mathrm{n}=36)$ y HDL bajo en un $12,5 \%(\mathrm{n}=9)$. De acuerdo a la clasificación de Fredrickson, en el 22,2\% ( $n=16)$ se observó un fenotipo IIa, un fenotipo IIb en 18\% $(n=13)$ y un fenotipo $\mathrm{V}$ en $8,1 \%(n=13)$ (tabla 1$)$.

\section{Efecto de la normalización de TSH y T4L sobre el} perfil lipídico y sus alteraciones

A la normalización del perfil tiroideo, se encontraron concentraciones de TSH de $1,4 \mu \mathrm{IU} / \mathrm{mL}$ (RIC 0,8-2,8) y T4L de $1,4 \mathrm{ng} / \mathrm{dL}$ (RIC 1,2-1,6). El tiempo en el que se logró la normalización del perfil tiroideo fue de 12 semanas (RIC 10-13). En el perfil lipídico, se evidenciaron diferencias estadísticamente significativas en las concentraciones de CT $[184 \mathrm{mg} / \mathrm{dL}$ (RIC 92-283) vs $147 \mathrm{mg} / \mathrm{dL}$ (RIC 92-220); $\mathrm{p}=0,05]$, LDL-C [99 mg/dL (RIC 44-150) vs $82 \mathrm{mg} / \mathrm{dL}$ (RIC 41-168); $\mathrm{p}=0,02]$ y TG [113 mg/dL (RIC 50-382) vs $88 \mathrm{mg} /$ dL (RIC 16-243); $\mathrm{p}=0,03$ ]. No se encontró diferencia en las concentraciones de HDL-C [48 mg/dL (RIC 2280) vs $47 \mathrm{mg} / \mathrm{dL}$ (RIC 24-75); p = 0,45] (figura 1). Se evidenció una reducción del $65 \%$ en la frecuencia de dislipidemia ( $\mathrm{p}=0,001), 42 \%$ en hipercolesterolemia $(p=0,03)$ y $65 \%$ en hipertrigliceridemia $(p=0,001)$. El

Tabla 1. Fenotipos de dislipidemia de acuerdo a la clasificación de Fredrickson antes del tratamiento con levotiroxina y a la normalización del perfil tiroideo

\begin{tabular}{cccc}
\hline Dislipidemia & $\begin{array}{c}\text { Antes del tratamiento con levotiroxina; } n \\
(\%)\end{array}$ & $\begin{array}{c}\text { A la normalización del perfil tiroideo; } \\
n(\%)\end{array}$ & $p=$ \\
\hline Tipo I & 0 & 0 & 0,01 \\
Tipo Ila & $16(22,2)$ & $4(5,6)$ & 0,01 \\
Tipo IIb & $13(18)$ & $2(2,8)$ & - \\
Tipo III & 0 & 0 & - \\
Tipo IV & 0 & $10(16,6)$ & 0,82 \\
Tipo V & $13(18,1)$ & 0 & - \\
\hline
\end{tabular}

Tipo de Dislipidemia: Fredrickson DS, Lees RS. A system for phenotyping hyperlipoproteinemia. Circulation 1965;31:321-7. doi: 10.1161/01.cir.31.3.321

Figura 1. Parámetros del perfil lipídico antes del tratamiento con levotiroxina y a la normalización del perfil tiroideo. *significancia estadística. LDL-C: COlesterol de baja densidad; HDL-C: colesterol de alta densidad; TG: trigliceridos; $\mathrm{CT}$ : colesterol total.

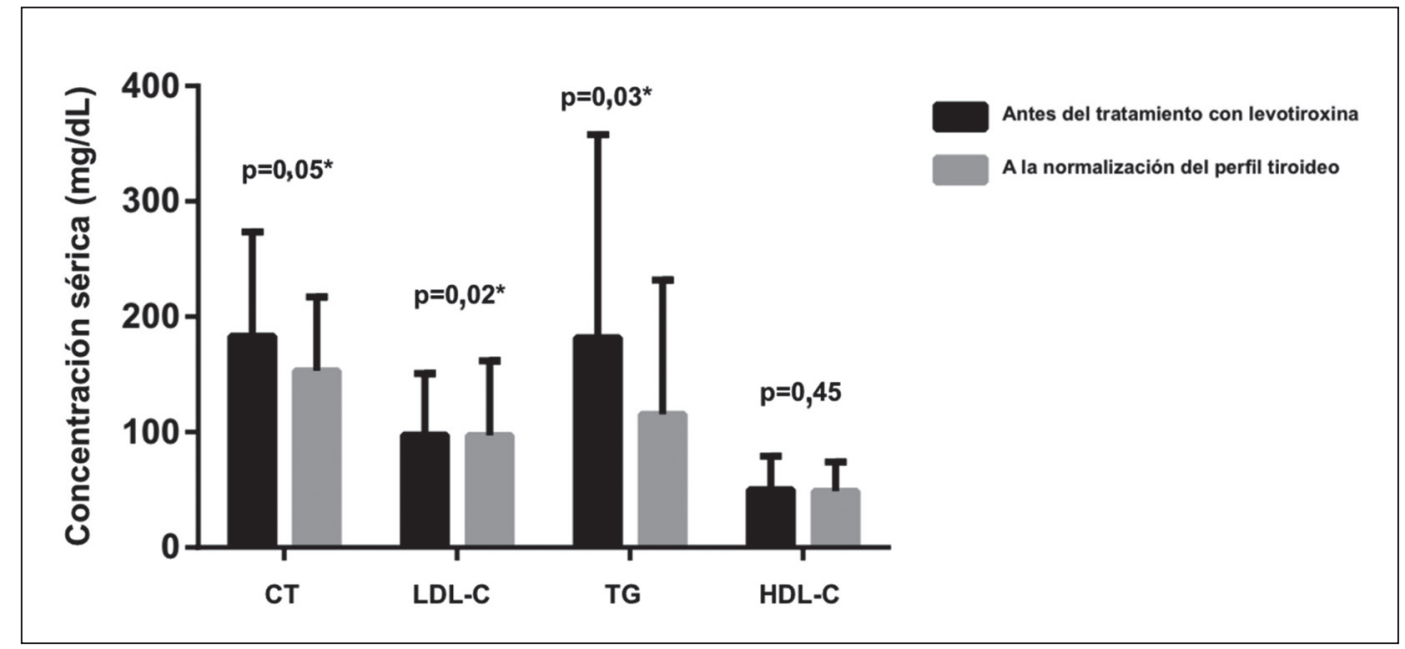


$22,2 \%(n=16)$ persistió con alteraciones del perfil lipídico, destacando hipercolesterolemia en $25 \%(n=18)$, hipertrigliceridemia en $12,5 \%(n=9)$ y aumento de LDL-C en $25 \%(\mathrm{n}=18)$, así como una mayor frecuencia de HDL bajo [12,5\% ( $\mathrm{n}=9)$ vs 26,3\% ( $\mathrm{n}=19)$; $\mathrm{p}=0,03$ ] (figura 2). De acuerdo a la clasificación de Fredrickson, se evidenció una reducción en la frecuencia del fenotipo IIa y IIb durante el tratamiento con levotiroxina (tabla 1 ).

\section{Asociación entre TSH, T4L, IMC y perfil lipídico antes del tratamiento con levotiroxina $y$ a la normalización del perfil tiroideo}

Al evaluar la asociación entre TSH, T4L y el perfil lipídico antes del tratamiento con levotiroxina, las concentraciones de TSH tuvieron una correlación positiva con la edad $(\mathrm{r}=0,39 ; \mathrm{p}=0,001)$, CT $(\mathrm{r}=0,36$; $\mathrm{p}=0,002)$ y LDL-C $(\mathrm{r}=0,46 ; \mathrm{p}=0,01)$, y una correlación negativa con HDL-C $(\mathrm{r}=-0,33 ; \mathrm{p}=0,004)$ y T4L $(r=-0,45 ; p=0,001)$. La concentración de T4L mostró una correlación positiva con HDL-C $(r=0,28$; $\mathrm{p}=0,02)$, aunado a una correlación negativa con LDL$\mathrm{C}(\mathrm{r}=-0,28 ; \mathrm{p}=0,02)$ y TSH $(\mathrm{r}=-0,45 ; \mathrm{p}=0,001)$. No se evidenció correlación entre TSH o T4L con el IMC y TG. Al evaluar la asociación entre IMC y el perfil lipídico, se demostró una correlación positiva entre IMC y TG $(r=0,39 ; p=0,001)$, sin evidencia de correlación con HDL-C, CT o LDL-C.

A la normalización del perfil tiroideo, no se evidenció asociación entre TSH y los parámetros del perfil lipídico. Las concentraciones de CT post-tratamiento se correlacionaron con CT pretratamiento $(r=0,40$; $\mathrm{p}=0,001)$ y LDL-C $(\mathrm{r}=0,34 ; \mathrm{p}=0,004)$ pretratamiento, así como con LDL-C $(r=0,88 ; \mathrm{p}=0,01)$ y con TG $(r=0,35 ; p=0,02)$ a la normalización del perfil tiroideo. En el caso de HDL-C, las concentraciones se correlacionaron de manera negativa con las de T4L $(\mathrm{r}=-0,27 ; \mathrm{p}=0,02), \mathrm{IMC}(\mathrm{r}=-0,42 ; \mathrm{p}=0,001)$ y TG $(\mathrm{r}=-0,38 ; \mathrm{p}=0,01)$ y las concentraciones de TG mostraron una correlación positiva con IMC $(\mathrm{r}=0,47$; $\mathrm{p}=0,001)$ y TG pre-tratamiento $(\mathrm{r}=0,50 ; \mathrm{p}=0,001)$. No se evidenció correlación de IMC con TSH, CT o LDL-C. La persistencia de dislipidemia a la normalización de TSH y T4L se asoció con la presencia de obesi$\operatorname{dad}(r=0,27 ; p=0,02)$, sobrepeso $(r=0,58 ; p=0,001)$ $\mathrm{y}$ dislipidemia pretratamiento $(\mathrm{r}=0,53 ; \mathrm{p}=0,001)$.

Dada la frecuencia encontrada de sobrepeso y obesidad, aunado a la asociación de TG y HDL-C con IMC, se compararon las características del perfil lipídico y tiroideo entre pacientes con y sin obesidad/sobrepeso. Antes del tratamiento con levotiroxina, no se observaron diferencias estadísticamente significativas en las concentraciones de TSH, T4L, CT, LDL-C, HDL-C y TG, sin embargo, a la normalización del perfil tiroideo, se evidenciaron mayores concentraciones de TG y menores de HDL-C en pacientes con obesidad/sobrepeso, sin diferencias para el resto de las variables (tabla 2).

\section{Discusión}

En el presente estudio reportamos el efecto de la normalización de TSH y T4L sobre el perfil lipídico y sus alteraciones en pacientes pediátricos con hipotiroidismo primario tratados con levotiroxina, así como la asociación de TSH y T4L con las concentraciones de CT, LDL-C y HDL-C antes del tratamiento con levotiroxina.

Las alteraciones en el metabolismo de lipoproteínas han sido reportadas hasta en el 52\% de adultos con hipotiroidismo primario ${ }^{11}$. En niños y adolescentes con hipotiroidismo, si bien se han descrito alteraciones en las concentraciones séricas de CT, TG, LDL-C, VLDL$\mathrm{C}$ y HDL-C, incluso en pacientes con hipotiroidismo

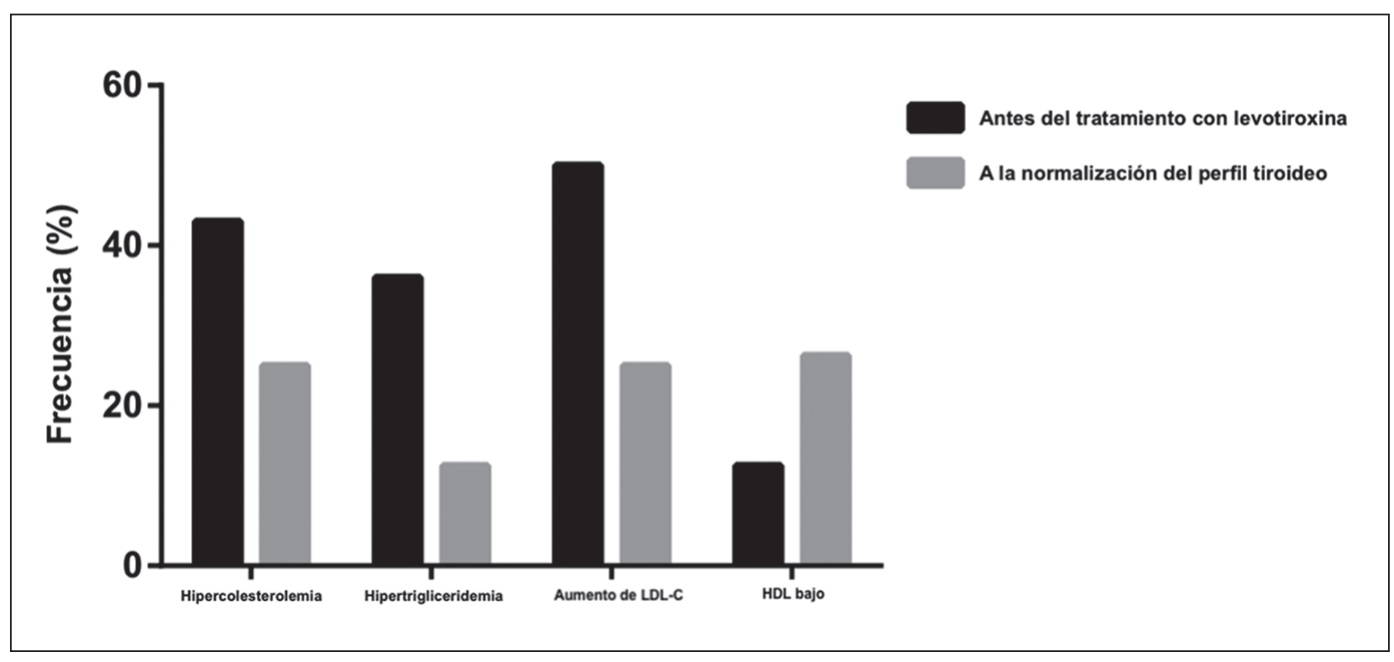

Figura 2. Frecuencia de dislipidemia antes del tratamiento con levotiroxina y a la normalización del perfil tiroideo. 
Tabla 2. Características del perfil lipídico, TSH y T4L en pacientes con y sin obesidad/sobrepeso

\begin{tabular}{|c|c|c|c|}
\hline & Sin obesidad/sobrepeso & Obesidad/sobrepeso & $\mathrm{p}$ \\
\hline \multicolumn{4}{|c|}{ Antes del tratamiento con levotiroxina } \\
\hline Colesterol total (mg/dL) & $200(157-222)$ & $169(144-210)$ & 0,18 \\
\hline $\mathrm{LDL}-\mathrm{C}(\mathrm{mg} / \mathrm{dL})$ & $124(89-141)$ & $95(74-144)$ & 0,31 \\
\hline $\mathrm{HDL}-\mathrm{C}(\mathrm{mg} / \mathrm{dL})$ & $50(44-60)$ & $45(41-53)$ & 0,07 \\
\hline Triglicéridos (mg/dL) & $96(78-189)$ & $114(83-176)$ & 0,55 \\
\hline $\mathrm{TSH}(\mu \mathrm{IU} / \mathrm{mL})$ & $38,8(18,5-77)$ & $21,2(12-47)$ & 0,14 \\
\hline T4L (ng/dL) & $0,6(0,4-0,7)$ & $0,49(0,3-0,6)$ & 0,20 \\
\hline \multicolumn{4}{|c|}{ A la normalización del perfil tiroideo } \\
\hline Colesterol total (mg/dL) & $154(126-175)$ & $144(128-170)$ & 0,5 \\
\hline $\mathrm{LDL}-\mathrm{C}(\mathrm{mg} / \mathrm{dL})$ & $82(66-96)$ & $82(63-105)$ & 0,88 \\
\hline $\mathrm{HDL}-\mathrm{C}(\mathrm{mg} / \mathrm{dL})$ & $50(45-56)$ & $42(39-49)$ & 0,006 \\
\hline Triglicéridos (mg/dL) & $74(61-112)$ & $112(65-162)$ & 0,02 \\
\hline $\mathrm{TSH}(\mu \mathrm{IU} / \mathrm{mL})$ & $1,0(0,6-2)$ & $1,3(0,9-2,12)$ & 0,06 \\
\hline T4L (ng/dL) & $1,4(1,2-1,6)$ & $1,3(1,2-1,6)$ & 0,99 \\
\hline
\end{tabular}

Variables cuantitativas reportadas en medianas y rango intercuartílico. LDL-C: colesterol de baja densidad, HDL-C: colesterol de alta densidad, TSH: hormona tiroestimulante, T4L: T4 libre.

subclínico $^{19}$, no se ha determinado una frecuencia de dislipidemia en general. En nuestra serie, la frecuencia evidenciada fue mayor al 50\%, semejante a la reportada en población no pediátrica. En hipotiroidismo se han descrito los fenotipos II y IV de dislipidemia ${ }^{18}$. En nuestro estudio, al utilizar la clasificación de Fredickson, encontramos un predominio del fenotipo IIa (LDL-C elevado, TG y VLDL normales, relación CT/ TG $>1,5$ ) y IIb (aumento de LDL-C, TG y VLDL) ${ }^{18}$, destacando un componente de dislipidemia mixta.

Los efectos del hipotiroidismo en el metabolismo de las lipoproteínas están relacionados con la disminución de las hormonas tiroideas ${ }^{4-10}$. En niños y adolescentes sin enfermedad tiroidea, se ha evidenciado una asociación positiva entre TSH y colesterol no$\mathrm{HDL}^{20}$.

En grupos específicos, como en pacientes con obesidad, se ha evaluado la asociación de TSH, perfil lipídico e IMC. En pacientes con obesidad sin diagnóstico de hipotiroidismo, se demostró una asociación positiva de TSH con CT y LDL-C, sin evidencia de asociación entre TSH e IMC ${ }^{21}$. En un estudio que incluyó pacientes con obesidad, tanto con hipotiroidismo subclínico como sin alteración tiroidea, se evidenció una asociación positiva de TSH con CT, TG e IMC y una asociación negativa de T4L y CT ${ }^{22}$. En nuestro estudio, durante el hipotiroidismo, evidenciamos la asociación positiva de TSH con CT y LDL-C y de T4L con HDL, así como la asociación negativa de TSH con HDL-C y T4L con LDL-C, sin corroborarse una asociación entre TSH e IMC. No evidenciamos asociación entre TG y TSH o T4L a diferencia del resto de los componentes del perfil lipídico. Con estos datos, demostramos la re- lación entre los parámetros del perfil tiroideo y lipídico en pacientes pediátricos con hipotiroidismo.

Diversos estudios han evidenciado la mejoría del perfil lipídico después del tratamiento con levotiroxina tanto en adultos ${ }^{11-14}$, como en población pediátri$\mathrm{Ca}^{23}$. En adultos con hipotiroidismo autoinmune se ha evidenciado la reducción de TG, apolipoproteína B (apoB) e índice aterogénico después del tratamiento con levotiroxina ${ }^{11}$. En adultos con hipotiroidismo post-quirúrgico, el tratamiento con levotiroxina demostró una reducción en las concentraciones de CT, TG, LDL-C, apolipoproteína A-I y apoB ${ }^{12}$.

Danese y cols, en una revisión sistemática, reportaron la disminución de las concentraciones de CT y LDL-C después del tratamiento con levotiroxina, con resultados no concluyentes para HDL-C y TG' ${ }^{13}$. En pacientes con hipotiroidismo subclínico también se ha evidenciado la reducción de CT y LDL-C posttratamiento ${ }^{14}$. En niños con hipotiroidismo subclínico, Cerbone y cols, observaron un aumento del índice aterogénico y de la relación TG/HDL-C, hiperhomocisteinemia y disminución de HDL-C ${ }^{24}$. Al evaluar el efecto de levotiroxina en este grupo de pacientes, se evidenció una disminución en la relación cintura-talla, TG/HDL-C e índice aterogénico, así como un aumento de HDL-C ${ }^{23}$. En otro estudio realizado en niños con hipotiroidismo subclínico, no se evidenciaron cambios en el perfil lipídico al lograrse el eutiroidismo ${ }^{25}$.

En nuestro estudio, evidenciamos una mejoría en las concentraciones de CT, TG y LDL-C a la normalización del perfil tiroideo, concordando con los hallazgos reportados en población adulta. Así mismo, evidenciamos una disminución en la frecuencia de 
dislipidemia. Es necesario señalar que, a pesar de los cambios evidenciados en el perfil lipídico a la normalización de TSH y T4L, más del 20\% de los pacientes persistió con dislipidemia, predominando la elevación de LDL y HDL bajo. Al eutiroidismo, corroboramos la asociación de CT con TG, CT pretratamiento y LDLC pre y post-tratamiento, de TG con IMC, de HDL-C con IMC y de la persistencia de dislipidemia con obesidad, sobrepeso y dislipidemia pretratamiento.

Estos hallazgos demuestran que, a pesar de la normalización del perfil tiroideo, existen otros factores asociados a dislipidemia persistente en este grupo de pacientes, tales como las alteraciones del estado nutricional (sobrepeso y obesidad) y la dislipidemia pretratamiento, siendo importante la intervención terapéutica oportuna de los mismos aunado al tratamiento con levotiroxina.

Un punto a destacar es la frecuencia de sobrepeso $[25 \%(n=18)]$ y obesidad $[27,8 \%(n=20)]$ encontrada en nuestro estudio, la cual es mayor a la reportada en la población pediátrica de acuerdo a la Encuesta Nacional de Salud en México (sobrepeso de 18,4\% y obesidad de $19,5 \%$ en población entre 5 y 11 años; sobrepeso de $24,7 \%$ y obesidad de $15 \%$ entre 12 y 19 años $)^{26}$. Si bien, no encontramos diferencias en las características del perfil lipídico pretratamiento, observamos mayores concentraciones de TG y menores de HDL-C en pacientes con obesidad/sobrepeso al eutiroidismo, lo cual, aunado a la correlación demostrada entre IMC, TG y HDL, refleja el papel de la obesidad y el sobrepeso en la dislipidemia persistente.

Dentro de las limitaciones del estudio destacan la naturaleza retrospectiva de su diseño, la falta de determinaciones bioquímicas de subunidades o fracciones de lipoproteínas, de marcadores de riesgo cardiovascular, así como la falta de control de variables como dieta y actividad física. Dentro de las fortalezas, destacamos el ser uno de los primeros estudios que evalúan el efecto de la normalización del TSH y T4L sobre el perfil lipídico en población pediátrica proveniente de un país latinoamericano con una alta prevalencia de enfermedades cardiometabólicas. Consideramos como futuras líneas de investigación la realización de estudios prospectivos para evaluar el efecto del eutiroidismo sobre el perfil lipídico y otros marcadores de riesgo cardiovascular.

En conclusión, la dislipidemia secundaria está presente en más de la mitad de los pacientes pediátricos con hipotiroidismo primario por tiroiditis de Hashimoto, existiendo una asociación entre TSH, T4L y el perfil lipídico. A la normalización del perfil tiroideo, existe una reducción de las concentraciones de CT, TG y LDL-C, así como de la frecuencia de dislipidemia. La dislipidemia persistente a pesar del logro del eutiroidismo está asociada a obesidad, sobrepeso y dislipidemia durante el hipotiroidismo, destacando la importancia de la intervención oportuna de estos factores aunado al tratamiento con levotiroxina.

\section{Responsabilidades Éticas}

Protección de personas y animales: Los autores declaran que los procedimientos seguidos se conformaron a las normas éticas del comité de experimentación humana responsable y de acuerdo con la Asociación Médica Mundial y la Declaración de Helsinki.

Confidencialidad de los datos: Los autores declaran que han seguido los protocolos de su centro de trabajo sobre la publicación de datos de pacientes.

\section{Derecho a la privacidad y consentimiento informa-} do: Los autores han obtenido el consentimiento informado de los pacientes y/o sujetos referidos en el artículo. Este documento obra en poder del autor de correspondencia.

\section{Conflicto de intereses}

Los autores declaran no tener conflicto de intereses.

\section{Referencias}

1. Duntas LH, Brenta G. The effect of thyroid disorders on lipid levels and metabolism. Med Clin North Am 2012;96(2):269-81. doi: 10.1016/j. mcna.2012.01.012.

2. Cappa M, Bizzarri C, Crea F. Autoimmune thyroid diseases in children. J Thyroid Res 2011:675703. doi: 10.4061/2011/6 75703 .

3. Rade Vukovic, Aleksandra Zeljkovic, Biljana Bufan, Vesna SpasojevicKalimanovska, Tatjana Milenkovic,
Jelena Vekic. Hashimoto Thyroiditis and Dyslipidemia in Childhood: A Review. Front Endocrinol (Lausanne) 2019;10:868. doi: 10.3389/ fendo.2019.00868.

4. Choi JW, Choi HS. The regulatory effects of thyroid hormone on the activity of 3-hydroxy-3-methylglutaryl coenzyme A reductase. Endocr Res 2000; 26(1):1-21. doi: 10.1080/07435800009040142.

5. Shin DJ, Osborne TF. Thyroid regulation and cholesterol metabolism are connected through sterol regulatory element-binding protein-2 (SREBP-2). J Biol Chem 2003;
278(36):34114-8. doi: 10.1074/jbc. M305417200.

6. Galman C, Bonde Y, Matasconi M, Angelin B, Rudling M. Dramatically increased intestinal absorption of cholesterol following hypophysectomy is normalized by thyroid hormone. Gastroenterology 2008;134:1127-36. doi: 10.1053/j.gastro.2008.01.032.

7. Tan KC, Shiu SW, Kung AW. Plasma cholesteryl ester transfer protein activity in hyper and hypothyroidism. J Clin Endocrinol Metab 1998; 83(1):140-3. doi: $10.1210 /$ jcem.83.1.4491. 
8. Lam KS, Chan MK, Yeung RT. Highdensity lipoprotein cholesterol, hepatic lipase and lipoprotein lipase activities in thyroid dysfunction - effects of treatment. Q J Med 1986;59:513-21.

9. Boone LR, Lagor WR, Moya Mde L, Niesen MI, Rothblat GH, Ness GC. Thyroid hormone enhances the ability of serum to accept cellular cholesterol via the ABCA1 transporter. Atherosclerosis 2011;218:77-82. doi: 10.1016/j. atherosclerosis.2011.04.028.

10. Bonde $Y$, Breuer O, Lütjohann D, Sjöberg $\mathrm{S}$, Angelin B, et al. Thyroid hormone reduces PCSK9 and stimulates bile acid synthesis in humans. J Lipid Res 2014;55(11):2408-15. doi: 10.1194/jlr. M051664.

11. Minarikova Z, Gaspar L, Kruzliak P, Celecova Z, Oravec S. The effects of treatment on lipoprotein subfractions evaluated by polyacrylamide gel electrophoresis in patients with autoimmune hypothyroidism and hyperthyroidism. Lipids Health Dis 2014;13:158. doi: 10.1186/1476-511X-13158.

12. Jung KY, Ahn HY, Han SK, Park YJ, Cho BY, Moon MK. Association between thyroid function and lipid profiles, apolipoproteins, and high-density lipoprotein function. J Clin Lipidol 2017;11(6):1347-53. doi: 10.1016/j. jacl.2017.08.015.

13. Danese MD, Ladenson PW, Meinert CL, Powe NR. Clinical review 115: effect of thyroxine therapy on serum lipoproteins in patients with mild thyroid failure: a quantitative review of the literature. J Clin Endocrinol Metab 2000;85(9):2993-01. doi: $10.1210 /$ jcem.85.9.6841.

14. Xiang Li, Yupeng Wang, Qingbo Guan, Jiajun Zhao, Ling Gao. The Lipid-
Lowering Effect of Levothyroxine in Patients With Subclinical Hypothyroidism: A Systematic Review and Meta-Analysis of Randomized Controlled Trials. Clin Endocrinol (Oxf) 2017;87(1):1-9. doi: 10.1111/cen.13338.

15. Barlow SE. Expert Committee. Expert committee recommendations regarding the prevention, assessment, and treatment of child and adolescent overweight and obesity: summary report. Pediatrics 2007;120 Suppl 4:S164-92. doi: 10.1542/ peds.2007-2329C.

16. US Preventive Services Task Force, Bibbins-Domingo K, Grossman DC, Curry SJ, Davidson KW, Epling JW Jr, et al. Screening for Lipid Disorders in Children and Adolescents: US Preventive Services Task Force Recommendation Statement. JAMA 2016;316(6):625-33. doi: 10.1001/jama.2016.9852.

17. National Cholesterol Education Program (NCEP): highlights of the report of the Expert Panel on Blood Cholesterol Levels in Children and Adolescents. Pediatrics 1992;89:495-501.

18. Fredrickson DS, Lees RS. A system for phenotyping hyperlipoproteinemia. Circulation 1965;31:321-7. doi: 10.1161/01.cir.31.3.321.

19. Dahl AR, Iqbal AM, Lteif AN, Pittock ST, Tebben PJ, et al. Mild subclinical hypothyroidism is associated with paediatric dyslipidaemia. Clin Endocrinol 2018;89(3):330-5. doi: 10.1111/cen.13752.

20. Witte T, Ittermann T, Thamm M, Riblet NB, Völzke H. Association between serum thyroid-stimulating hormone levels and serum lipids in children and adolescents: a population-based study of german youth. J Clin Endocrinol Metab 2015;100(5):2090-7. doi: 10.1210/jc.2014.

21. Aeberli I, Jung A, Murer SB, et al. During rapid weight loss in obese children, reductions in TSH predict improvements in insulin sensitivity independent of changes in body weight or fat. J Clin Endocrinol Metab. 2010;95(12):5412-8. doi:10.1210/jc.2010-1169.

22. Jin HY. Prevalence of subclinical hypothyroidism in obese children or adolescents and association between thyroid hormone and the components of metabolic syndrome. J Paediatr Child Health. 2018;54(9):975-80. doi:10.1111/ jpc.13926.

23. Cerbone M, Capalbo D, Wasniewska M, Alfano S, Mattace Raso G, et al. Effects of L-thyroxine treatment on early markers of atherosclerotic disease in children with subclinical hypothyroidism. Eur J Endocrinol 2016;175(1):11-9. doi: 10.1530/EJE-15-0833.

24. Cerbone M, Capalbo D, Wasniewska M, Mattace Raso G, Alfano S, et al. Cardiovascular Risk Factors in Children With Long-Standing Untreated Idiopathic Subclinical Hypothyroidism. J Clin Endocrinol Metab 2014;99(8):2697-703. doi: 10.1210/jc.2014-1761

25. Çatlı G, Anık A, Ünver Tuhan H, Böber E, Abacı A. The effect of L-thyroxine treatment on hypothyroid symptom scores and lipid profile in children with subclinical hypothyroidism. J Clin Res Pediatr Endocrinol 2014;6(4):238-44. doi: 10.4274/Jcrpe. 1594.

26. Instituto Nacional de Salud Pública Instituto Nacional de Estadística y Geografía. Encuesta Nacional de Salud y Nutrición 2018. Presentación de resultados, México, https://ensanut. insp.mx/encuestas/ensanut2018/doctos/ informes/ensanut_2018_presentacion_ resultados.pdf; 2019 [acceso el 4 de agosto de 2020]. 